\title{
Alterstice
}

Revue internationale de la recherche interculturelle

International Journal of Intercultural Research

Revista International de la Investigacion Intercultural

\section{Accueillir les élèves migrants : dispositifs et interactions à l'école publique en France}

\section{Isabelle Rigoni}

Volume 7, numéro 1, 2017

L'interculturel dans la Cité : actes à poser en contexte pluriethnique

URI : https://id.erudit.org/iderudit/1040610ar

DOI : https://doi.org/10.7202/1040610ar

Aller au sommaire du numéro

Éditeur(s)

Alterstice

ISSN

1923-919X (numérique)

Découvrir la revue

Citer cet article

Rigoni, I. (2017). Accueillir les élèves migrants : dispositifs et interactions à l'école publique en France. Alterstice, 7(1), 39-50.

https://doi.org/10.7202/1040610ar

\section{Résumé de l'article}

La France est un vieux pays d'immigration accueillant des familles depuis la fin du $19^{\mathrm{e}}$ siècle. Depuis, les flux migratoires n'ont cessé de se diversifier et de se complexifier. Le défi est de taille pour les travailleurs sociaux, mais également pour l'institution scolaire, qui doit intégrer chaque année plusieurs dizaines de milliers d'élèves migrants. Si l'école française est obligatoire pour tous les enfants depuis 1882, les aménagements pour les élèves allophones arrivants sont une disposition relativement récente. Comment penser l'accueil scolaire de ces enfants et de ces jeunes, inscrits parfois dans des contextes paupérisés et stigmatisés et dans lesquels les enjeux sociaux, politiques et politiques sont déjà nombreux? Comment également penser les tensions politiques qui entourent les questions migratoires, et quelles en sont les conséquences pour les élèves et les personnes qui les accompagnent? Cet article propose une analyse des rapports qui se nouent entre différents acteurs de l'éducation en interaction (enseignants, intervenants sociaux et éducatifs) et la structuration des expériences éducatives d'enfants et jeunes migrants. Il s'agit d'analyser la capacité des acteurs professionnels à apporter un appui ajusté et pertinent aux difficultés et aux exigences auxquelles les enfants et jeunes sont soumis dans des cadres éducatifs normatifs et contraignants. Nous nous appuyons sur les résultats de plusieurs programmes de recherche visant, au moyen d'entretiens semi-directifs mais aussi d'observations et de méthodes collaboratives et participatives, à mieux connaître les conditions effectives d'accueil et de scolarisation offertes par les institutions scolaire et d'intervention sociale, afin d'appréhender les fonctionnements institutionnels au regard des attentes des familles et de leurs enfants, d'analyser les expériences migratoire et scolaire des enfants et jeunes et enfin de saisir les dimensions pluri-professionnelles de l'accompagnement éducatif pour ces populations.
Ce document est protégé par la loi sur le droit d'auteur. L'utilisation des services d'Érudit (y compris la reproduction) est assujettie à sa politique d'utilisation que vous pouvez consulter en ligne.

https://apropos.erudit.org/fr/usagers/politique-dutilisation/ 


\section{7}

alterstice

Revue Internationale

de la Recherche Interculturelle

مevista International

de la Investigacion Intercultural

ARTICLE THÉMATIQUE

\section{Accueillir les élèves migrants : dispositifs et interactions à l'école publique en France}

Isabelle Rigoni ${ }^{1}$

\section{Résumé}

La France est un vieux pays d'immigration accueillant des familles depuis la fin du $19^{\mathrm{e}}$ siècle. Depuis, les flux migratoires n'ont cessé de se diversifier et de se complexifier. Le défi est de taille pour les travailleurs sociaux, mais également pour l'institution scolaire, qui doit intégrer chaque année plusieurs dizaines de milliers d'élèves migrants. Si l'école française est obligatoire pour tous les enfants depuis 1882, les aménagements pour les élèves allophones arrivants sont une disposition relativement récente. Comment penser l'accueil scolaire de ces enfants et de ces jeunes, inscrits parfois dans des contextes paupérisés et stigmatisés et dans lesquels les enjeux sociaux, politiques et politiques sont déjà nombreux? Comment également penser les tensions politiques qui entourent les questions migratoires, et quelles en sont les conséquences pour les élèves et les personnes qui les accompagnent? Cet article propose une analyse des rapports qui se nouent entre différents acteurs de l'éducation en interaction (enseignants, intervenants sociaux et éducatifs) et la structuration des expériences éducatives d'enfants et jeunes migrants. II s'agit d'analyser la capacité des acteurs professionnels à apporter un appui ajusté et pertinent aux difficultés et aux exigences auxquelles les enfants et jeunes sont soumis dans des cadres éducatifs normatifs et contraignants. Nous nous appuyons sur les résultats de plusieurs programmes de recherche visant, au moyen d'entretiens semi-directifs mais aussi d'observations et de méthodes collaboratives et participatives, à mieux connaître les conditions effectives d'accueil et de scolarisation offertes par les institutions scolaire et d'intervention sociale, afin d'appréhender les fonctionnements institutionnels au regard des attentes des familles et de leurs enfants, d'analyser les expériences migratoire et scolaire des enfants et jeunes et enfin de saisir les dimensions pluri-professionnelles de l'accompagnement éducatif pour ces populations.

\section{Rattachement de l'auteure \\ ${ }^{1}$ Institut d'enseignement supérieur et de recherche Handicaps et besoins éducatifs particuliers (INS HEA), Équipe Ghrapes, Suresnes, France}

\section{Correspondance}

isabelle.rigoni@inshea.fr

\section{Mots clés}

migrations; réfugiés; école

\section{Pour citer cet article}

Rigoni, I. (2017). Accueillir les élèves migrants : dispositifs et interactions à l'école publique en France. Alterstice, 7(1), 39-50. 


\section{Introduction}

Comme d'autres pays d'immigration en Europe et en Amérique du Nord, la France, depuis la fin du $19^{\mathrm{e}}$ siècle, accueille des familles (Jablonka, 2010), dont les enfants sont massivement scolarisés à l'école publique. Chaque année, l'institution scolaire doit ainsi intégrer plusieurs dizaines de milliers d'élèves primo-migrants, composant un public hétérogène plus ou moins familier des habitudes scolaires, de la culture et de la langue françaises. Pourtant, si l'obligation scolaire est entrée en vigueur pour tous les enfants avec la loi Ferry de $1882^{1}$, il faut attendre un siècle et les vagues migratoires concomitantes des Trente Glorieuses pour voir naître des aménagements destinés aux élèves allophones arrivants, soit à partir des années 1970. Dans le même temps, au Royaume-Uni et aux ÉtatsUnis, le concept de special educational needs (besoins éducatifs particuliers, BEP) fait son apparition, et il sera repris plus tard en France. Aujourd'hui, le débat tant national qu'international porte sur la notion d'inclusion, pour faire des élèves à BEP des membres à part entière de l'école.

Cependant, rares sont les études qui ont traité du public spécifique des élèves allophones arrivants, tant en matière de contenu et de pratiques pédagogiques que d'analyse des orientations des élèves à la sortie, comme le déplorent à la fois chercheurs (Abdallah-Pretceille et Barbot, 2003; Schiff, 2003) et institutions (OCDE, 2006). On sait cependant, grâce aux enquêtes PISA de 2003 et 2013 qui évaluent les compétences des élèves dans 65 pays, que les résultats scolaires des élèves allochtones sont inférieurs à ceux des autochtones, avec des écarts importants en France, où l'école ne parvient pas à lutter suffisamment contre les déterminismes sociaux et les inégalités scolaires (Caille et Rosenwald, 2006 ; Dubet, Duru-Bellat et Vérétout, 2010). Dans ce contexte, comment (re)penser l'accueil scolaire des jeunes migrants, inscrits parfois dans des contextes paupérisés et stigmatisés dans lesquels les enjeux sociaux, économiques et politiques sont déjà nombreux?

Par ailleurs, on relève des contradictions entre les politiques inclusives volontaristes du ministère de l'Éducation nationale et les pratiques du ministère de l'Intérieur à l'encontre des enfants dont les familles sont en situation irrégulière, voire des mineurs isolés étrangers (Senovilla Hernandez, 2013). Si l'École a pour obligation d'accueillir tous les mineurs de moins de 16 ans, français ou étrangers, en situation légale ou non, les pratiques d'expulsion d'élèves et de leurs familles perdurent. Dénoncées depuis plus de dix ans par le Réseau Éducation Sans Frontière (RESF), elles ont parfois fait la une des médias et constitué un enjeu politique national, par exemple avec l'« affaire Leonarda $»^{2}$. Quelles sont les conséquences de ces tensions politiques pour les élèves et les personnes qui les accompagnent?

Cet article présente une analyse des rapports qui se nouent entre différents acteurs de l'éducation en interaction (enseignants, intervenants sociaux et éducatifs), influençant la structuration des expériences éducatives d'enfants et de jeunes migrants. II s'agit d'analyser la capacité des acteurs professionnels à apporter un appui ajusté et pertinent aux difficultés et aux exigences auxquelles les enfants et les jeunes sont soumis dans des cadres éducatifs normatifs et contraignants, et ce, grâce à l'étude des interrelations entre ces différents intervenants éducatifs aux logiques et aux pratiques professionnelles parfois différentes. Pour ce faire, nous nous appuyons sur les résultats de plusieurs programmes de recherche ${ }^{3}$ que nous coordonnons, en mettant en lumière premièrement les conditions effectives d'accueil et de scolarisation de la part des institutions scolaire et d'intervention sociale, afin de comprendre les fonctionnements institutionnels au regard des attentes et expériences des familles et des jeunes, deuxièmement l'expérience scolaire des élèves allophones arrivants et troisièmement les dimensions pluriprofessionnelles de l'accompagnement éducatif des élèves allophones arrivants.

\footnotetext{
${ }^{1}$ Ministre de l'Instruction Publique Jules Ferry, Loi n 11696 du 28 Mars 1882, Article 4, Journal Officiel du 29 mars 1882.

${ }^{2} L^{\prime}$ ' affaire Leonarda » a connu un fort retentissement médiatique suite à l'expulsion vers le Kosovo, le 9 octobre 2013, d'une famille rom immigrée dont une fille mineure et collégienne, Leonarda Dibrani, a été interpellée lors d'une sortie scolaire.

3 MIGRITI, financé par I'Université Paris Lumières (2014-2015) (http://migriti.hypotheses.org), EVASCOL, financé par le Défenseur des Droits (2015-2017) (http://evascol.hypotheses.org), SAJE, financé par la Maison des Sciences de l'Homme et de la Société de Poitiers (2015-2016) et ALTERECOLE, financé par la Région Aquitaine (2016-2018).
} 
Les projets de recherche dans lesquels s'inscrit ce travail qualitatif portent sur plusieurs académies ${ }^{4}$, permettant ainsi la comparaison. Mais le terrain investigué par nos soins concerne plus spécifiquement l'agglomération bordelaise. D'une part, nous nous appuyons sur des entretiens menés auprès d'une vingtaine d'acteurs institutionnels relevant de l'Éducation nationale ainsi que des municipalités qui ont à leur charge d'inscrire les élèves du premier degré et de gérer les écoles élémentaires ${ }^{5}$. D'autre part, une place prépondérante a été accordée à l'observation sur site, dans plusieurs dispositifs spécifiques de trois établissements du premier et du second degrés, qui scolarisent les élèves de 6 à 15 ans. Chaque dispositif a été observé lors d'une demi-journée hebdomadaire pendant quinze à vingt semaines. Lors de ces séances, nous avons développé, en collaboration avec les enseignants et des intervenants artistiques extérieurs, des méthodes collaboratives et participatives adaptées pour recueillir la parole d'enfants et d'adolescents, en se démarquant d'une posture adulto-centrée (Armagnague et Rigoni, 2016). Nous avons également interrogé 72 élèves et rencontré une dizaine de parents, avec lesquels nous avons échangé sur leurs projets et leurs attentes.

\section{Conditions d'accueil et de scolarisation des élèves migrants}

Les conditions d'accueil et de scolarisation des élèves migrants ont évolué au cours du $20^{\mathrm{e}}$ siècle et jusqu'à récemment. Dans ce processus, plusieurs types d'acteurs institutionnels sont présents aux niveaux national et local, et ont une influence sur les trajectoires des élèves et les attentes des familles.

\section{Les dispositifs spécifiques, une réponse institutionnelle bientôt centenaire}

L'offre éducative à destination des élèves migrants a largement évolué depuis près d'un siècle en France, à partir d'une focale longtemps centrée sur le retour des familles et les liens culturels et linguistiques avec les pays d'origine. Dès 1925, des cours de langue vivante portant également sur l'histoire et la géographie du pays d'origine commencent à être assurés dans les écoles françaises, par des professeurs étrangers, en dehors du temps scolaire, à l'intention des enfants issus de l'immigration. Cette période marque les débuts de l'enseignement des langues et cultures d'origine (ELCO), favorisées par les États d'émigration qui y voient le moyen de garder un lien fort avec leur diaspora, ainsi que par les institutions supranationales dans le but de garantir le maintien de la diversité des langues et cultures.

D'autres types de dispositifs sont consacrés à l'accueil des élèves migrants, pendant le temps scolaire cette fois. Ils prennent place au moment de l'âge d'or de l'immigration, pendant les Trente Glorieuses et juste avant I'institutionnalisation du regroupement familial en 1976. Ces dispositifs conçus pour accompagner la scolarisation des " enfants étrangers non francophones " prennent d'abord la forme de classes d'insertion en 1970 (classes d'initiation pour non-francophones, CLIN) puis de classes d'adaptation pour le secondaire en 1973 (classes d'adaptation, CLAD). Ces classes d'accueil sont dites "fermées » dans la mesure où les jeunes qui y sont scolarisés ne vont pas en classe ordinaire. En 1976, la création des Centres de formation et d'information pour la scolarisation des enfants de migrants (CEFISEM) chargés de valoriser les "cultures d'origine " et de promouvoir des pratiques pédagogiques " adaptées » consacrent une politique différentialiste en la reliant à la mauvaise conscience coloniale et postcoloniale (Sayad, 2014). L'apparition de ces dispositifs s'inscrit également dans un contexte marqué par l'écho que rencontre un ensemble de discours issus des mouvements pédagogiques, de l'Éducation nouvelle, puis du tournant critique post-68, qui tentent de (re)définir de nouvelles méthodes et de nouveaux contenus pour l'école en prenant en compte différentes catégories de populations.

Désormais, les préoccupations de l'Éducation nationale commencent à être liées à l'émergence des problématiques liées aux besoins éducatifs particuliers et à l'inclusion. Le concept de besoins éducatifs particuliers (BEP) a été conçu en Angleterre dans les années 1975-1978 et développé par Seamus Hegarty et Mary Warnock sous le terme de special educational needs, avant d'être adopté aux États-Unis. En moins de vingt ans, celui-ci a été instauré dans nombre de pays en tant que socle de la scolarisation de tous les élèves en difficulté, qu'elle soit durable,

\footnotetext{
${ }^{4}$ Académies de Bordeaux, de Créteil, de Montpellier et de Strasbourg.

${ }^{5}$ Le premier degré concerne les écoles maternelles (3-6 ans) et élémentaires (6-11 ans) et sont gérées par les mairies; le second degré comprend les collèges (11-15 ans) gérés par les départements et les lycées (15-18 ans) gérés par les régions. La scolarisation est obligatoire de 6 à 16 ans.
} 
permanente ou passagère. Cette évolution s'accompagne d'un changement de paradigme de l'insertion ou de l'intégration au profit de l'inclusion. Promue par les institutions supranationales au cours des années 1990, l'inclusion résulte d'un mouvement mobilisant acteurs associatifs et chercheurs autour d'un modèle social refusant l'exclusion des personnes au profit de l'acceptation de leur différence. Ce mouvement s'oppose ainsi à toute forme de scolarisation en milieu spécialisé ou en dispositif ségrégatif susceptible d'enfermer les individus dans une logique de filière stigmatisante et vulnérabilisante ainsi qu'à toute forme de scolarisation se satisfaisant d'une intégration de l'élève à BEP, faisant de ceux-ci des élèves qui sont « dans l'école » mais qui ne sont pas membres " de l'école » (Ebersold, 2009). Partant du principe que la scolarisation de tout élève est profitable à tous, l'école est appelée à développer un sens de la communauté et un soutien mutuel. L'inclusion se rapporte ainsi aux termes de justice sociale et d'égalité des chances (Dubet, 2004, 2010), avec le principe que le système scolaire doit s'adapter aux besoins particuliers plutôt que l'inverse.

En France, toutefois, les cloisonnements conceptuels et réglementaires se maintiennent longtemps. II faut attendre plusieurs circulaires en $2012^{6}$ pour que l'Éducation nationale réforme les CLIN et les CLAD au profit des UPE2A et des UPS, destinés respectivement aux " élèves allophones nouvellement arrivés » (EANA) et aux " enfants des familles itinérantes et de voyageurs" (EFIV), et pour que les missions des Centres académiques pour la scolarisation des EANA et des EFIV (CASNAV), héritiers des CEFISEM, soient redéfinis. Les jeunes bénéficiant de ces récents dispositifs sont inscrits en classe ordinaire et intègrent I'UPE2A ou I'UPS quelques heures quotidiennes ou hebdomadaires, en fonction de leurs besoins. Pour le ministère, l'inclusion dans les classes ordinaires doit constituer la modalité principale de scolarisation. Quelques éléments sont avancés pour permettre de favoriser l'inclusion, tels que la personnalisation des parcours pour permettre à l'élève de suivre un enseignement dans sa classe ordinaire en fonction des compétences qu'il a acquises antérieurement et de son degré de maîtrise de la langue française ; la concertation et la formation des équipes pour organiser les liens avec la classe ordinaire, mieux comprendre les processus d'apprentissage pour ces élèves et ainsi adapter les contenus d'enseignement; le suivi des élèves dans la durée pour favoriser leur inclusion dans le système scolaire français et enfin l'information aux familles pour les associer à la scolarisation de leur enfant.

Toutefois, nos enquêtes montrent que le fonctionnement de ces dispositifs diffère sensiblement d'une académie à une autre, d'une école à une autre, d'une équipe éducative à l'autre. L'ouverture des dispositifs au profit d'une plus grande inclusion dépend à la fois des pratiques culturelles des acteurs institutionnels locaux, des établissements scolaires et des professionnels dont les profils, la formation tout autant que la situation dans l'école et dans la classe diffèrent parfois fortement selon les territoires.

\section{Récents courants migratoires, ajustements institutionnels et projets individuels}

Les pratiques inclusives doivent également s'adapter à l'hétérogénéité des flux migratoires. L'impact des conflits et des désordres régionaux sur les courants migratoires est bien connu. Mais les bouleversements engendrés par les révolutions arabes, le conflit syrien et plus globalement les déséquilibres moyen-orientaux ont des conséquences importantes en matière de flux migratoires, comme en témoignent le nombre inédit de migrants et de réfugiés tentant de traverser la mer Méditerranée ${ }^{7}$. Si la France se distingue d'autres pays d'Europe occidentale par son faible taux d'absorption de réfugiés, les pouvoirs publics doivent néanmoins ajuster leur politique d'accueil. La plupart des réfugiés arrivent par leurs propres moyens, mais plusieurs centaines d'entre eux sont envoyés chaque mois par la Grèce ou l'Italie, conformément au programme européen de "relocalisation » validé à l'été 2015 qui prévoit que la France accueille 30000 réfugiés sur un objectif européen total de 160000 personnes. Les pouvoirs publics y voient l'objectif de justifier une politique de répartition des migrants et des réfugiés sur l'ensemble du territoire national, déjà entamée depuis plusieurs années et encore récemment accentuée avec le démantèlement

\footnotetext{
${ }^{6}$ Circulaires n 2012-141, 2012-142 et 2012-143 parues au Bulletin Officiel n 37 du 11 octobre 2012.

7 Près d'un million de migrants et réfugiés ont traversé la mer Méditerranée en 2015 (contre 219000 en 2014) selon le Haut Commissariat des Nations-Unies pour les réfugiés (HCR) et 3700 y ont perdu la vie; en 2016, c'est le cas de près de 5000 . De plus, l'Organisation internationale des migrations (OIM) estime que plus de 34000 migrants et réfugiés se sont rendus en 2015 en Bulgarie et en Grèce après avoir traversé la Turquie. " Le total représente le flux migratoire le plus élevé depuis la Seconde guerre mondiale » en Europe, précise l'OIM dans une note aux médias ("Le cap du million de migrants arrivés en Europe en 2015 a été franchi », Le Monde, 22 décembre 2015).
} 
de la « jungle » de Calais depuis le 29 février 2016. Comme ceux « relocalisés » en provenance de Grèce ou d'Italie, les migrants et réfugiés quittant Calais ou Dunkerque, volontairement ou non, sont répartis, au mieux, dans des centres d'accueil de demandeurs d'asile (CADA) sur l'ensemble du territoire national. Les enfants des familles qui y sont accueillies sont scolarisés dans des écoles de secteur qui, souvent, incluent un dispositif spécifique de type UPE2A. Depuis l'afflux de réfugiés syriens en Europe et le programme de "relocalisation », les services académiques se sont préparés, localement, l'arrivée des élèves réfugiés. Toutefois, au printemps puis à l'automne 2016, nombre de migrants et de réfugiés "relocalisés » étaient envoyés dans des centres d'accueil et d'orientation sans que la scolarisation de leurs enfants ne soit assurée. Acteurs politiques, sociaux et éducatifs sont mobilisés en fonction de leurs intérêts propres et les contradictions ne manquent pas entre, d'une part, des préfectures parfois tentées d'associer réussite scolaire des enfants et droit au séjour des familles et, d'autre part, des associations faisant valoir le droit à l'éducation ou à la protection; ou encore entre des préfectures qui envoient des familles de demandeurs d'asile à plusieurs centaines de kilomètres de leur lieu de résidence, et des équipes éducatives qui dénoncent de ce fait la discontinuité scolaire des enfants ainsi re-déplacés.

De même, les mineurs isolés étrangers (MIE) induisent de nouvelles préoccupations et de nouveaux questionnements sur les plans politique et juridique, mais également sur le terrain scolaire (Nora-Roger-Vasselin, 2015) et en termes d'accompagnement (Kobanda Ngbenza, 2016). Leur nombre est grandissant en France où ils sont estimés à 8000 dans l'hexagone et autant dans les départements et territoires d'outre-mer. Juridiquement mineurs et séparés de leurs représentants légaux, les MIE sont en situation d'isolement et ne disposent pas d'un statut juridique propre (Senovilla Hernandez, 2013). Ils se trouvent à un croisement, relevant à la fois du droit des étrangers et, au titre de l'enfance en danger, du dispositif français de protection de l'enfance, qui ne pose aucune condition de nationalité. Localement, les conseils départementaux sont chargés de s'occuper des MIE au titre de l'aide sociale à l'enfance (ASE) dont les missions comprennent le soutien éducatif et à l'orientation. Comme dans le cas des réfugiés et des demandeurs d'asile, les tensions sont constantes entre, d'une part, les pouvoirs publics garants des politiques d'accueil et de séjour restrictives et, d'autre part, les acteurs éducatifs, sociaux et associatifs œuvrant en faveur des droits de l'enfant et du droit à la scolarisation. Comme les réfugiés et les demandeurs d'asile, les MIE sont dispersés dans différents départements selon une politique de "péréquation ", pour être placés dans des foyers et des centres d'accueil et ainsi éviter l'errance juvénile telle qu'elle se pratique notamment en région parisienne. Toutefois, cette politique induit un report de la scolarisation effective, voire dans certains cas des processus de déscolarisation-rescolarisation.

Face à ces contraintes, les projets et les attentes des jeunes et de leur famille doivent s'adapter. Les entretiens avec les élèves et avec les parents montrent l'hétérogénéité des causes migratoires, que ce soit la fuite des conflits armés et du manque de sécurité dans le pays d'origine, comme dans le cas de familles syriennes, irakiennes, yéménites et ukrainiennes rencontrées; la fuite des conditions économiques difficiles, comme les familles des pays du sud de l'Europe qui remontent vers le sud-ouest de la France, ou les familles originaires d'Afrique ayant résidé plusieurs années en Espagne, en Italie ou au Portugal et qui migrent une nouvelle fois; la recherche de soins adaptés pour un membre de la famille malade, ainsi que cette élève albanaise de 8 ans venue en France pour se faire soigner; ou encore la volonté d'apprendre le français à son enfant. Plus ou moins conscients, selon leur âge, de ces projets migratoires et des contraintes institutionnelles auxquelles les familles doivent faire face, les enfants n'en vivent pas moins des expériences migratoires et scolaires individuelles significatives.

\section{Expériences migratoires et scolaires des enfants et des jeunes}

En 2014-2015, 52500 élèves allophones étaient répartis dans près de 9200 écoles et collèges, représentant 0,56 \% des élèves des premier et second degrés ${ }^{8}$. À noter toutefois que certains élèves ne sont pas repérés ou comptabilisés, tandis que d'autres sont déscolarisés. Leur répartition varie fortement selon les académies, allant de plus de 7000 élèves allophones dans celle de Versailles à quelques centaines dans quelques académies rurales ou dans les départements d'outre-mer hors Guyane. En prenant comme l'exemple l'académie de Bordeaux, nous nous

\footnotetext{
${ }^{8}$ Minisère de l'Éducation nationale, Division de l'Évaluation, de la Prospective et de la Performance (DEPP), Note d'information, $\mathrm{n}^{\circ} 35$, octobre 2015.

http://cache.media.education.gouv.fr/file/2015/79/2/depp-ni-2015-35-eleves-allophones-2014-2015_486792.pdf
} 
référons à un territoire où la présence migratoire est ancienne, historiquement marquée par le commerce colonial puis par l'immigration en provenance des pays d'Europe du Sud, avant que de nouveaux courants migratoires arrivent de l'est de l'Europe, en particulier de Turquie puis de Bulgarie. Avec un taux de répartition des élèves allophones qui la situe dans la moyenne des académies hors Île-de-France, les dispositifs UPE2A qu'elle abrite constituent de riches laboratoires pour appréhender les expériences tant migratoires que scolaires.

En dehors des entretiens semi-directifs que nous avons effectués auprès des élèves en fin d'année scolaire après une période d'observation et de mise en confiance de plusieurs mois ${ }^{9}$, plusieurs méthodes collaboratives nous ont permis de recueillir, tout au long de l'année scolaire, la parole des enquêtés à propos de leurs expériences migratoires et scolaires. Dans le premier degré, les entretiens compréhensifs ou les récits de vie sont difficilement opératoires avec des enfants (6-10 ans) qui, en outre, ne maîtrisent pas, ou très peu, la langue française. Dans le second degré, les jeunes (10-15 ans) sont souvent embarrassés par la relation de face-à-face que suppose l'entretien, qui rappelle parfois un rapport de domination élève-enseignant ou jeune-adulte pouvant jouer un rôle inhibant ou promouvoir une attitude de faux semblant (Goffman, 1975/1963). D'autant plus lorsque se superposent le traumatisme de migrations douloureuses et la précarité administrative, économique et sociale. Avec eux tous, nous avons donc préféré concevoir des méthodes créatives et collaboratives visant à les placer en situation de co-production voire d'analyse de nos résultats, à l'instar de quelques rares expérimentations anthropologiques dans le cadre scolaire (Mariano Longo et Roche, 2015). Nous avons ainsi réalisé, sur chaque site, la constitution de séances construites expressément pour l'enquête, souvent en collaboration avec les enseignants intervenant dans les dispositifs (réalisation de carnets de voyage, de boîtes à souvenirs, d'une émission de radio sur le thème de la migration) voire avec des intervenants extérieurs (écriture d'un scénario et réalisation d'un court métrage sur la diversité et l'accueil, saynètes de théâtre-forum sur l'accueil des migrants à l'école, réalisation d'un espace partagé dans un quartier populaire) qui ont permis, grâce à l'expression libre, de recueillir un riche matériau sur les expériences des jeunes dans leurs parcours personnel et scolaire.

\section{L'expérience migratoire exprimée dans le cadre scolaire}

L'un des constats les plus marquants est celui de la forte hétérogénéité des parcours migratoires, en fonction des pays de départ et des ressources des familles. Certaines d'entre elles arrivent en France par avion, d'autres en bus ou en voiture après plusieurs jours de route, d'autres encore dans des conditions qu'elles ne souhaitent pas exprimer. Rares sont les familles qui migrent ensemble, l'un des parents venant avec tout ou une partie des enfants (" on a menti à ma sœur pour qu'elle ne vienne pas », élève portugais, 7 ans), des enfants arrivant avec un autre membre de leur famille (" je suis venue avec une dame et mon petit frère », élève géorgienne, 11 ans), la plupart laissant des êtres chers ("En Russie, je vivais dans une ferme avec mes parents, mes frère et sœurs et mes grandsparents. Mes grands-parents sont restés en Russie ", élève russe, 8 ans) et certains autres arrivant seuls et gonflant les rangs des mineurs isolés étrangers, parfois placés dans des foyers et accompagnés par des éducateurs, parfois contraints de rester dans la rue. Si les raisons de migrer sont multiples pour les parents, les enfants n'en ont pas toujours conscience car celles-ci n'ont pas toujours été exprimées, et certains refusent le projet migratoire de leurs parents (" je n'aime pas ma vie ici, je veux retourner en Grèce », collégien grec, 15 ans). En dépit de l'hétérogénéité des parcours et des situations, il est ainsi fréquent de recenser des expériences douloureuses, en premier lieu celle de la séparation avec des membres de la famille ou, parfois, avec des amis.

Certaines familles ont migré à plusieurs reprises, comme celles en provenance de pays subsahariens ou du Maghreb, ayant transité plusieurs années en Espagne, au Portugal ou en Italie où ils ont parfois acquis la nationalité, avant de rejoindre l'agglomération bordelaise du fait de la crise économique et financière dans les pays du sud de l'Europe. Scolarisés dans les différents pays, les enfants sont souvent plurilingues mais ont parfois des difficultés à se repérer dans le temps et dans l'espace lorsque ces migrations successives ont eu lieu au cours de la jeune enfance.

\footnotetext{
${ }^{9}$ Seule exception, quelques extraits d'entretiens cités dans cet article ont été menés par Mélanie Jaubert, dans le cadre de son stage d'études dans une UPE2A du second degré.
} 
Parfois, les familles sont transnationales (Razy et Baby-Collin, 2011) : " Je n'ai pas de famille ici mais en Angleterre " (élève ukrainienne, 10 ans) ou " Je suis venu en France avec mes parents et ma grand-mère, tout le reste de ma famille est resté en Italie, mais j'ai aussi de la famille en Afrique » (élève italien d'origine africaine, 11 ans). Dans ces cas, il semble que la migration soit mieux acceptée ou mieux comprise par les enfants, qui n'en font qu'un constat dénué d'amertume ou d'inquiétude. Certains élèves expriment aussi leur satisfaction d'avoir rejoint une partie de leur famille: "J'ai beaucoup de famille en France, je me sens très très bien ici, ma famille est ici » (collégienne marocaine, 11 ans).

Du côté des élèves n'ayant pas connu la migration, la volonté d'accueillir un camarade ne parvenant pas bien à s'exprimer et à comprendre la langue française n'est pas toujours non plus une expérience aisée : " Ça m'ennuie d'accueillir / J'ai la flemme d'aller accueillir / J'ai envie d'être tranquille / Ils restent en groupe / Ils ne veulent pas se mélanger " (extraits de préparation aux séances de théâtre-forum avec la compagnie Digame, en collaboration avec l'association Promofemmes, classe de CM2 d'une école du centre-ville de Bordeaux). Lors d'entretiens classiques, ces sentiments auraient été plus difficilement dicibles. Dans le cadre du théâtre-forum, ils permettent de poursuivre l'expression directe sur le thème de la (non)mixité par les enfants et jeunes migrants: "Pourquoi on ne se mélange pas? / Parce qu'on n’a pas envie / Pourquoi on n’a pas envie ? / Parce qu'on a peur » (idem).

\section{L'expérience scolaire après la migration}

L'expérience de la scolarisation est nouvelle pour certains élèves qui n'ont jamais été à l'école dans leur pays d'origine, soit parce que le système scolaire n'impose aucune obligation avant l'âge de 7 ans, soit en raison de conflits armés, soit par crainte de certaines familles envers le système scolaire et les institutions en général comme dans le cas de familles roms ou itinérantes. Si la plupart de ces nouveaux élèves sont envoyés dans des dispositifs spécifiques pour les non scolarisés antérieurement (UPE2A-NSA), certains d'entre eux arrivent dans les dispositifs UPE2A classiques, soit par manque de dispositifs adaptés ou par manque de place dans les dispositifs existants, soit du fait de la dissimulation par les parents de l'absence de scolarisation antérieure de leur enfant. Dans chacun des dispositifs étudiés, les enseignants ont fait mention d'un ou de plusieurs cas d'élèves non scolarisés antérieurement, en décalage par rapport au groupe d'élèves qui, pour leur part, disposaient déjà de certains habitus scolaires. Cette situation engendre des difficultés tant pour les élèves qui doivent prendre le temps d'acquérir des codes communs que pour les enseignants qui doivent orienter leurs apprentissages tant sur le savoir-être que sur les connaissances.

Pour l'ensemble des élèves cependant, l'école est un lieu de socialisation, ce qui se vérifie plus encore dans le cadre des situations interculturelles. La scolarisation est l'occasion d'appréhender les différences entre les cultures et les modes de socialisation. Plus que les relations avec l'équipe éducative, les élèves soulignent les relations amicales avec leurs pairs, immigrés ou non : " Ici, les enfants ne jouent pas dans la rue, mais à l'école on se fait des copains » (élève russe d'origine arménienne, 8 ans, non scolarisé antérieurement); " Je me suis fait des amis [au collège], on parle mi-anglais, mi-français " (collégien mexicain, 14 ans) ; "L'UPE2A aide à se faire des amis " (collégien tunisien, 13 ans). Le brassage des cultures et des points de vue induit par la scolarisation permet aussi, à l'inverse, de mettre des mots sur certaines formes de racisme ou de mise à l'écart. Certains élèves arrivent à l'école avec les images des autres transmises par leur entourage : hiérarchies, humiliations, préjugés - qu'ils en soient instigateurs ou victimes. Ainsi, lors d'une séance de discussion avec des parents d'élèves, un groupe de six femmes bulgares ont commencé à s'invectiver, les Bulgares turcophones accusant les autres de ne pas être comme elles parce que roms, tandis que la seule Bulgare non turcophone et non rom se tenait à l'écart de ces échanges. Le calme est revenu par l'entremise de l'interprète, une travailleuse sociale turcophone de Turquie ! Ailleurs, lors d'une séance de discussion avec des élèves, plusieurs ont exprimé des propos xénophobes tels que " je n'aime pas l'Afrique » (élève géorgienne, 11 ans) et "l'Afrique, ça schlingue! " (élève russe d'origine arménienne, 11 ans). Ces échanges sont le reflet de sociétés ethniquement très hiérarchisées dont le modèle de différenciation sociale est reproduit à l'école. C'est justement parce que la façon de faire société des enfants et jeunes ne peut pas être conçue comme un processus naturel, résultant de la somme de leurs spontanéités, que le rôle de l'équipe éducative est primordial, notamment dans ses choix pédagogiques. L'observation des relations entre pairs confirme les résultats récemment avancés par Mariano Longo et Roche à l'issue de leur travail dans deux classes d'écoles primaires en Roumanie et en Italie, selon lesquels 
les relations interculturelles apaisées sont favorisées lorsque « la pédagogie donne de l'importance à la diversité, à la solidarité et à l'ouverture de l'école sur le monde extérieur » (2015, p. 170).

L'école est également un lieu d'apprentissage et d'acquisition de connaissances et l'UPE2A met surtout l'accent sur la maîtrise de la langue française et des mathématiques, tout en s'appuyant sur les expériences des élèves pour aborder des matières comme la géographie. Dans le second degré, rares sont les élèves interrogés qui, comme ce collégien italien d'origine africaine de 11 ans, auraient préféré être totalement inclus en classe ordinaire dès leur arrivée : «Je n'aime presque rien dans I'UPE2A car on voit des choses que j'apprenais déjà en Italie. Je n'aime pas rester avec des gens qui ne sont pas de mon niveau, je suis le plus petit de l'UPE2A. [...] Je veux rester qu'avec les $6^{\mathrm{e}}$ [en classe ordinaire] car en UPE2A il y a des $3^{e}$ ". Les entretiens révèlent au contraire que beaucoup apprécient d'être dans I'UPE2A pour acquérir les notions de français, indispensables selon eux à pouvoir suivre les autres cours en classe ordinaire et, plus largement, à mieux participer à la vie sociale : "L'UPE2A m'aide beaucoup à apprendre le français et à m'intégrer " (collégienne kosovare, 12 ans). Certains avancent aussi le fait rassurant et confortable d'être avec d'autres élèves étrangers avec lesquels ils partagent des expériences similaires et peuvent s'exprimer en partie dans leur langue d'origine, ou en anglais. Surtout, on constate que plus la pédagogie respecte le rythme d'apprentissage de chaque élève, plus ceux-ci développent une confiance en soi leur permettant d'acquérir et de consolider leurs apprentissages. La temporalité à la carte des dispositifs UPE2A que nous avons étudiés, dans lesquels les élèves sont intégrés en fonction de leurs besoins individuels, permet souvent cette pédagogie différenciée, si tant est que les effectifs ne soient pas trop importants.

\section{Dimensions pluri-professionnelles de l'accompagnement éducatif}

La structuration des expériences scolaires des enfants et des jeunes migrants est également sous-tendue par les rapports qui se nouent entre les différents acteurs de l'éducation en interaction, tels que l'institution scolaire, les enseignants et les intervenants sociaux et éducatifs.

\section{Gérer les relations de travail dans des espaces à négocier}

La singularité des dispositifs d'accueil de type UPE2A complexifie les relations professionnelles en ajoutant un acteur central : I'enseignant référent. Particulièrement dans le second degré, les enseignants de classe ordinaire et celui relevant du dispositif doivent régulièrement négocier entre eux l'emploi du temps des élèves en fonction de leurs progrès linguistiques autant que de leur autonomie. En tant que dispositif ouvert, l'UPE2A accueille les élèves nécessitant un enseignement adapté en français langue seconde (FLS) mais, leurs besoins étant variables, chaque élève peut disposer d'un emploi du temps à la carte, particulièrement au collège, ce qui produit parfois de nombreuses allées et venues dans et hors du dispositif vers les classes ordinaires. Ces arrangements se règlent au sein de l'équipe éducative et peuvent parfois donner lieu à des frictions entre collègues. Les notions de frontière, d'identité professionnelle, de reconnaissance du travail de l'autre sont sensibles et se présentent dans des espaces à négocier.

Pour porter la réflexion sur les relations pluri-professionnelles, il est possible de s'inspirer des voies avancées dans le champ du travail social. Parmi elles, l'approche pluridisciplinaire permet d'apporter des éclairages différents, sans qu'il y ait pour autant croisement ou imbrication des savoirs ou des méthodes. Dans le champ scolaire, cette approche offre une large ouverture à travers l'échange entre les différents acteurs éducatifs, mais le risque est de multiplier les points de vue sur l'évolution de l'élève sans jamais pouvoir les croiser, au point d'aboutir à un empilement d'analyses, de remarques, de résultats. Aussi, pour essayer de favoriser la coopération professionnelle, la transdisciplinarité a été avancée pour tenter d'élaborer un savoir commun. Ici, les professionnels acceptent l'idée d'interchangeabilité et se centrent sur le projet de vie, sur les particularités individuelles. Mais c'est bien parce que " l'ouverture à la transdisciplinarité comporte l'acceptation de l'inconnu, de l'inattendu et de l'imprévisible » que cette démarche est difficile à mettre en œuvre (Nicolescu, 1996, p. 178). Au sein des équipes éducatives, les professionnels peuvent se sentir démunis ou mis en danger par une démarche qui les implique (trop) directement dans les projets des élèves qui, dans le cas des EANA, sont des projets non seulement scolaires mais également liés à la migration ou installation, tant ces aspects sont imbriqués. Les enseignants en UPE2A sont de facto rompus à un accompagnement des élèves migrants qui dépasse parfois le seul cadre scolaire pour déborder sur le social voire le juridique. Particulièrement dans le cas des élèves issus de familles en demande d'asile, certains enseignants de 
dispositifs apportent un soutien moral voire technique ou financier aux familles de leurs élèves les plus en difficulté - que ce soit par une simple bienveillance à l'égard d'élèves dont on sait qu'ils sont mal logés ou même à la rue, ou par une implication plus importante qui peut se traduire par l'organisation de collectes de fonds ou par une collaboration avec des associations de soutien à la scolarisation et au séjour. II n'en va pas de même des enseignants de classe ordinaire, souvent plus éloignés de ces préoccupations. Tous les enseignants ne sont donc pas interchangeables en matière d'accompagnement de l'élève, et ceux les mieux à même d'effectuer ce travail sont les enseignants d'UPE2A qui, bien souvent, ont une connaissance plus fine des difficultés intrinsèques à la migration et à l'installation des familles.

Il n'en demeure pas moins que les différentes composantes de l'équipe éducative doivent coopérer. Pour ce faire, l'approche interdisciplinaire telle qu'elle est conçue dans le champ du travail social semble la plus appropriée tant elle suppose dialogue et échange de connaissances, d'analyses, de méthodes entre différents professionnels. En favorisant l'existence d'interactions et d'enrichissement mutuel entre les enseignants, cette approche devrait permettre la mise en commun de connaissances et de perceptions sur les élèves migrants qui permettent à ces derniers d'évoluer favorablement au sein de l'institution scolaire et dans leurs apprentissages. Cette démarche interdisciplinaire apparaît comme une finalité nécessaire au travail en équipe afin de (re)placer l'élève comme auteur de son parcours et d'affirmer une cohésion des pratiques éducatives. Le contexte coopératif favorise ces échanges vers une co-construction d'une compréhension réciproque. Le recueil de l'expérience d'une enseignante en UPE2A illustre bien cette nécessité : « Ici, les relations sont très bonnes avec les collègues [de classe ordinaire] et je n'ai pas de difficulté à composer l'emploi du temps, à monter des projets avec les élèves... Mais dans l'académie [où j'enseignais précédemment], les relations étaient difficiles et nous avions du mal à travailler ensemble ", au point que les projets étaient peu ou pas existants tant ils étaient rendus complexes à mettre en œuvre, et le suivi des élèves migrants était mis à mal du fait des difficultés de communication et de coopération au sein même de l'équipe éducative.

Un travail d'équipe mené en bonne intelligence permet de mieux comprendre les parcours et les projets individuels et de garantir les conditions d'inclusion en contexte pluriethnique. Pour autant, on ne peut faire l'économie de précieux garde-fous relatifs aux postures professionnelles et à l'éthique dans le travail d'intervention auprès des populations migrantes. Comme plus largement dans le champ du travail social, l'acte à poser doit être établi avec prudence et l'accompagnement doit être mesuré. Quoi de plus paradoxal en effet que de défendre les vertus de l'accompagnement tout en adhérant à l'idée selon laquelle tout individu est autonome par nature ? Plusieurs enseignants travaillant avec des élèves migrants mais aussi avec des élèves issus de familles de voyageurs dénoncent le "grand maternage » comme l'effet pervers des dispositifs d'accueil en milieu scolaire : "il faut travailler sur l'autonomisation! ». Si l'injonction à l'autonomie est paradoxale dans le contexte éducatif, il ne faut pas moins en tenir compte. Ici, la régulation des relations entre l'élève et l'institution scolaire passe par la nécessité de faire intervenir ce que les travailleurs sociaux appellent un collectif d'accompagnement, composé de plusieurs membres de l'équipe éducative et destiné à co-esquisser des projets individualisés avec l'élève.

\section{Enseigner et se faire enseigner}

L'accompagnement éducatif pose également la question de l'enseignement, des lieux et des méthodes d'apprentissage. L'évolution des configurations institutionnelles avec le passage de dispositifs fermés (CLIN et CLAD) à des dispositifs ouverts (UPE2A) témoigne d'une prise en considération par les pouvoirs publics de la nécessité d'inclure les élèves à BEP dans le système scolaire. Toutefois, le paradigme de l'inclusion ne rencontre pas le même succès selon les territoires. Certaines académies, ou certains départements à forte concentration immigrée, peinent à mener à bien une politique inclusive des élèves primo-migrants et certains dispositifs UPE2A fonctionnent encore en circuit fermé, parfois par habitude ou par facilité. Par ailleurs, l'adéquation des dispositifs aux besoins sur les territoires est parfois obsolète. Les routes migratoires évoluent, les populations migrantes se renouvellent, les quartiers changent, certaines zones s'urbanisent et se densifient, pourtant " II manque une réflexion partagée entre l'Éducation nationale et la mairie sur l'urbanisme et ses effets; il faut s'interroger sur l'aménagement du territoire " (inspectrice de l’Éducation nationale). Plusieurs enseignantes déplorent "une explosion des effectifs depuis deux ans " sans que les moyens qui leur sont alloués n'augmentent; à l'inverse une autre se demande «quand décidera-t-on d'envoyer les enseignants là où il y a des élèves? Je vais dans la 
commune $[X]$ où il n'y a que dix élèves; ça fait trois ans que je le fais remonter à mon directeur de circonscription qui dit qu'on ne peut pas enlever de poste dans une école " alors que dans une commune voisine, les moyens font défaut. Le manque de souplesse de l'Institution entre en contradiction avec la fluidité des flux migratoires, plus encore lors de périodes d'afflux de réfugiés.

Par ailleurs, l'Éducation nationale se heurte également aux contrastes d'attractivité des territoires qui conduisent à des profils professionnels très différents au sein des équipes pédagogiques en charge de l'inclusion des élèves migrants. On recense des enseignants moins formés, plus précarisés et un turn-over plus important dans les zones paupérisées à forte concentration migratoire, souvent considérées comme des territoires ghettoïsés (Lapeyronnie, 2008) ou ségrégués (Van Zanten, 2001) abritant des " jeunes en survie " (Dubet, 1987). Au contraire, certaines académies plus attractives par leur qualité de vie et des zones urbaines aux relations sociales plus apaisées attirent des enseignants souvent mieux formés et titulaires de leur poste depuis de nombreuses années. Les incidences liées à ces contrastes d'attractivité sont notables car, en travaillant avec les élèves migrants, les professionnels doivent faire preuve de compétences additionnelles par rapport à celles attendues d'une équipe éducative. Or la formation des enseignants aux problématiques migratoires et aux relations interculturelles est extrêmement faible voire inexistante, même pour ceux qui font le choix d'enseigner en UPE2A. Seuls les CASNAV proposent une ou plusieurs journées de formation annuelles, mais les moyens varient encore selon les académies. De même, la plupart des enseignants rencontrés déplorent l'inexistence d'outils les aidant dans l'accompagnement scolaire des élèves, comme l'exprime cette enseignante, arrivée par hasard en dispositif UPE2A :

la problématique [des migrants] n'était pas du tout abordée pendant la formation en IUFM [anciens instituts de formation des enseignants, ancêtres des Espé] lorsque [j'étais] étudiante. J'ai appris sur le tas et aussi beaucoup avec ma collègue avec qui je travaille en binôme. En 2014, c'était une première, je suis intervenue auprès de tous les étudiants de l'Espé sur l'inclusion scolaire et la langue de scolarisation. [...] Avec ma collègue, nous avons une démarche de co-construction et nous expérimentons la fusion de nos groupes d'élèves dans nos deux UPE2A, ce qui conduit à une émulation et à une vraie bienveillance entre eux. Nous réfléchissons aussi à des outils pédagogiques adaptés.

En effet, ici et là, nous rencontrons des initiatives locales (au niveau académique, avec la constitution de mallettes pédagogiques) voire isolées (certains enseignants) sans que celles-ci soient centralisées ou systématisées. Les choix pédagogiques sont donc plutôt liés à ces initiatives locales et dépendantes de bonnes volontés sur le terrain.

Enfin, l'implication des familles, mise en avant par les institutions, dépend largement des dispositions prises par les chefs d'établissements ainsi que, plus largement, par l'équipe éducative. Certains enseignants, surtout dans le premier degré, prennent de leur temps personnel pour rencontrer les parents et essayer de les impliquer dans la vie scolaire. Mais les moyens accordés par les pouvoirs publics ne sont pas suffisants pour mettre en œuvre un accompagnement des familles primo-migrantes, souvent ignorantes du système scolaire français. Le cas des réunions parents-enseignants est emblématique, où les enseignants doivent trouver des ressources locales, dans l'établissement scolaire ou auprès des associations de quartier, pour assurer l'interprétariat. Dans le second degré, le dispositif "Ouvrir l'École aux parents pour la réussite des enfants " (OEPRE), conduit en partenariat entre le ministère de l'Intérieur et celui de l'Éducation nationale ${ }^{10}$, vise à favoriser l'intégration des parents d'élèves volontaires en leur dispensant un enseignement de la langue française ${ }^{11}$ ainsi que la connaissance des valeurs de la République et du fonctionnement et des attentes de l'École. Elles sont assurées majoritairement par des enseignants, essentiellement formés à l'enseignement du français langue seconde : des enseignants des UPE2A ou des professeurs possédant une certification en français langue seconde, en français langue étrangère ou ayant suivi une formation en français langue d'intégration. Elles sont également conduites par des professeurs d'histoiregéographie, de science et vie de la terre et de physique, quelques principaux ou directeurs d'école, des conseillers principaux d'éducation ou même des infirmières scolaires. Des interventions plus ponctuelles sont assurées par des personnels des réseaux d'écoute, d'appui et d'accompagnement des parents (REAAP), des personnels de santé, de personnels territoriaux ou de musées... Des personnels d'associations agréées par le ministère de l'Éducation

${ }^{10}$ Circulaire $n^{\circ} 2014-165$ du 14 novembre 2014.

${ }^{11}$ Des manuels ont récemment été publiés : Escouffier, Maric, Vérom, L'école ouverte aux parents. Apprendre le français pour accompagner la scolarité de ses enfants - Livret 1 \& 2 + Guide du formateur, Grenoble, Presses universitaires de Grenoble, 2014. Présentation en ligne : http://ecoleouverteauxparents.jimdo.com 
nationale, de la Jeunesse et de la vie associative ou prestataires de l'office français de l'immigration et de l'intégration (OFII) assurent également ces formations. Toutefois, ces dispositifs tournés vers les parents ne concernent qu'une faible part d'entre eux et, de plus, n'accueillent que des migrants hors Union européenne résidant en France de façon régulière depuis moins de cinq ans.

\section{Éléments de conclusion}

Les flux migratoires ne cessent de se renouveler, en particulier lors des périodes de crise économique et financière et lors des violences armées dans les pays d'origine, comme en témoigne la période contemporaine. Pour autant, l'arrivée d'enfants et de jeunes migrants demeure une constante, en dépit de l'hétérogénéité des modes d'entrée sur le territoire et des projets de vie. Qu'ils arrivent seuls ou en famille, de façon régulière ou non, tous ont droit à une scolarisation, et leurs expériences scolaires sont aussi diverses que leurs expériences migratoires. Les professionnels de l'éducation qui les accompagnent doivent ajuster leurs pratiques pédagogiques à ces élèves à besoins éducatifs particuliers que sont les élèves migrants, souvent allophones. Pourtant, les moyens alloués sont parfois faibles ou peu adaptés, et les contrastes territoriaux importants. À la lumière des expériences menées au niveau scolaire et éducatif, ainsi que du débat politique et social autour des questions interculturelles, il semble aujourd'hui impératif d'orienter la réflexion tant des acteurs scientifiques qu'institutionnels sur la question du respect et de la promotion du patrimoine linguistique et, surtout, du patrimoine culturel de nos sociétés contemporaines. Ceci, en posant les jalons d'une véritable politique globale d'éducation à l'interculturel et à l'interreligieux, qui passerait par toutes les matières enseignées aux élèves, mais aussi par la formation des équipes éducatives.

\section{Références bibliographiques}

Abdallah-Pretceille, M. et Barbot, J.-M. (2003). Le français comme langue étrangère et seconde. Dans L. Colles, J.--L. Dufays et C. Maeder (dir.), Enseigner le français, l'espagnol et l'italien - Les langues romanes à I'heure des compétences. Bruxelles : De Boeck.

Armagnague, M. et Rigoni, I. (2016). Saisir le point de vue de l'enfant. Enquêter sur la participation socio-scolaire des élèves migrants. Recherches qualitatives, Hors série 20 (Prudence empirique et risque interprétatif), 311-319. http://www.recherche-qualitative.qc.ca/documents/files/revue/hors_serie/HS-20/rq-hs-20armagnague-rigoni.pdf

Caille, J.-P. et Rosenwald, F. (2006). Les inégalités de réussite à l'école élémentaire : construction et évolution. Dans Ministère de l'Éducation nationale, de l'Enseignement supérieur et de la Recherche (dir.), France, portrait social. Paris : Ministère de l'Éducation nationale, de l'Enseignement supérieur et de la Recherche, Division de l'Évaluation, de la Prospective et de la Performance (DEPP).

Dubet, F. (1987). La galère : jeunes en survie. Paris : Fayard.

Dubet, F. (2004). L'école des chances. Paris : Seuil.

Dubet, F. (2010). Les places et les chances. Paris : Seuil.

Dubet, F., Duru-Bellat, M. et Vérétout, A. (2010). Les sociétés et leur école. Emprise du diplôme et cohésion sociale. Paris : Seuil.

Ebersold, S. (2009). Inclusion. Recherche et formation, 61, 71-83.

Goffman, E. (1975/1963). Stigmates. Les usages sociaux des handicaps. Paris : Minuit.

Jablonka, I. (2010). Les enfants de la République. L'intégration des jeunes de 1789 à nos jours. Paris : Seuil.

Kobanda Ngbenza, D. (2016). Enfants isolés étrangers. Une vie et un parcours faits d'obstacles. Paris : L'Harmattan, coll. Logiques sociales.

Lapeyronnie, D. (2008). Ghetto urbain. Ségrégation, violence, pauvreté en France aujourd’hui. Paris : Laffont.

Mariano Longo, T. et Roche, T. (2015). L'enfance à l'école des autres. Paris : Téraèdre. 
Nicolescu, B. (1996). La transdisciplinarité. Monaco : Éd. du Rocher.

Nora-Roger-Vasselin, M. (2015). Sexisation, ethnicisation et racisation dans et par l'action publique de scolarisation des mineurs isolés étrangers en tant qu'élèves allophones arrivant à Paris (Mémoire de maîtrise inédit). Université Paris Diderot.

Organisation de coopération et de développement économiques (OCDE) (2006) Points forts des élèves issus de l'immigration. Une analyse comparative des performances et de l'engagement des élèves dans PISA 2003. OCDE.

Razy, É. et Baby-Collin, V. (2011). La famille transnationale dans tous ses états. Autrepart, 57-58, 7-22.

Sayad, A. (2014). L'école et les enfants de l'immigration. Essais critiques. Paris : Seuil.

Schiff, C. (dir.). (2003). Non scolarisation, déscolarisation et scolarisation partielle des migrants. Paris : ministère de l’Éducation nationale, programme interministériel de recherche sur les processus de déscolarisation.

Senovilla Hernandez, D. (2013). Mineurs isolés étrangers et sans protection en Europe. Rapport final comparatif. Rapport de recherche de l'enquête PUCAFREU.

Van Zanten, A. (2001). L'école de la périphérie. Scolarité et ségrégation en banlieue. Paris : Presses universitaires de France. 